\title{
Téoros
}

Revue de recherche en tourisme

\section{La formation supérieure en tourisme offerte à distance ou en mode hybride}

\section{Essai sur des modèles innovants de gestion académique et de gestion administrative}

\section{Boualem Kadri et François Bédard}

Volume 27, numéro 2, été 2008

URI : https://id.erudit.org/iderudit/1070800ar

DOI : https://doi.org/10.7202/1070800ar

Aller au sommaire du numéro

Éditeur(s)

Université du Québec à Montréal

ISSN

0712-8657 (imprimé)

1923-2705 (numérique)

Découvrir la revue

Citer cette note

Kadri, B. \& Bédard, F. (2008). La formation supérieure en tourisme offerte à distance ou en mode hybride : essai sur des modèles innovants de gestion académique et de gestion administrative. Téoros, 27(2), 67-71.

https://doi.org/10.7202/1070800ar d'utilisation que vous pouvez consulter en ligne. 


\section{Centreinternational de formation et de recherche en tourisme \\ La formation supérieure en tourisme offerte à distance ou en mode hybride \\ Essai sur des modèles innovants de gestion académique et de gestion administrative}

\section{Boualem Kadri et François Bédard}

La formation à distance ou en mode hybride (présentiel et à distance) représente pour l'université des enjeux économiques (positionnement des institutions dans le marché international de la formation) et pédagogiques (refondation de la relation traditionnelle enseignant-apprenant). Toutefois, la problématique de la transformation du mode de création et de diffusion du savoir par les nouvelles technologies ne se réduit pas aux dimensions économique, pédagogique et technologique, mais concerne aussi l'aspect organisationnel. Or la recherche scientifique semble s'intéresser davantage à la dimension pédagogique. Certes, les enjeux organisationnel et administratif sont reconnus comme importants (CREPUQ, 1999 ; Karsenti et Larose, 2001), mais peu d'information et d'analyses sont disponibles et elles se réduisent souvent à quelques observations. Les dimensions administrative et organisationnelle de la formation à distance ou en mode hybride ont besoin d'être renforcées et investies autant que les dimensions pédagogique et technologique. Comment observer ces dimensions administrative et organisationnelle ? Cette réflexion sur la gestion académique est entreprise dans le cadre d'un projet du Fonds de développement académique du réseau de I'Université du Québec (FODAR) réalisé conjointement par l'UQAM, la TÉLUQ (l'Université à distance de I'UQAM) et I'ETS (École de technologie supérieure) ${ }^{1}$. Ce projet s'articule autour de trois orientations spécifiques : l'appropriation d'une instrumentation technologique, l'adaptation des pratiques pédagogiques de formation de cycles supérieurs et l'adaptation des pratiques de gestion académique. Les résultats préliminaires liés à ce troisième aspect font l'objet de cette chronique.

\section{L'UQAM et la formation à distance ou en mode hybride}

L'université, pôle important de la société où se construit l'innovation, fait face à des défis et à des enjeux importants (administratifs, pédagogiques, didactiques, culturels et éducatifs) entourant le problème de l'intégration des TIC (technologies de l'information et de la télécommunication) et dont on a une nette prise de conscience (Karsenti et Larose, 2001). À I'UQAM, de nombreuses interventions ont marqué le processus d'adaptation des TIC à la formation et à la recherche, notamment durant la décennie 19962006 : enquêtes après des étudiants (1996-1999) ; analyse des attitudes et des comportements des enseignants (2002 et 2005)². Sur le plan stratégique, on observe l'amorce d'une vision du développement des TIC à l'université. Ainsi, après une période de foisonnement d'initiatives individuelles menées par des professeurs et des chargés de cours, le besoin de concertation et d'intégration des actions pousse à la création, en 1997, d'un comité conseil du Vice-rectorat aux services académiques et au développement technologique (VRSADT). Un premier plan TI (Plan d'action pour l'intégration des technologies de l'information dans la formation) pour la période 1998-2001 voit le jour avec l'objectif de mettre en place une structure centrale - le CFITP (Centre de formation et d'innovations technopédagogiques) -, dans le but de former les enseignants et de soutenir leurs projets visant l'adaptation des TIC à la formation. Cette action est suivie en 2002 par la décentralisation de certaines activités du CFITP vers les facultés. Les centres d'initiatives facultaires (CIF) sont nés, étape cruciale dans le processus d'adaptation et d'intégration des TIC et outil majeur d'appropriation des technologies dans la formation. L'évaluation du fonctionnement des CIF - engagée chaque année depuis 2003 et qui a pris fin en 2005 - a eu une signification particulière, compte tenu de l'institutionnalisation des CIF dans le fonctionnement. Cette mesure a amorcé une étape nouvelle dans le processus d'intégration et d'appropriation des TIC, celle de la mise en réseau des CIF, qui devait être effective en 2006. Par ailleurs, le document préparatif du plan TI 2003-2006 prenait en compte les perspectives de rattachement de la TÉLUQ et capitalisait sur « les acquis de I'UQAM en ligne » (UQAM-VRAE $\left.{ }^{3}, 2003\right)$.

Après être passée du stade de premier cercle d'initiés à celui de la masse critique d'utilisateurs, I'UQAM estime avoir atteint un autre stade de développement où les questions d'organisation et de partage d'activités entre les services centraux et les facultés et entre les facultés elles-mêmes revêtent tout autant d'importance que les questions technologiques et pédagogiques. Par ailleurs, le rattachement de la TÉLUQ à l'UQAM ayant été enclenché, les centres d'initiatives facultaires devaient être des lieux de réalisation de projets pilotes touchant la formation à distance, la constitution de banques d'objets d'apprentissage ainsi que le développement de modèles de formation bimodaux. Les CIF devaient être un élément important de l'opération de rattachement de la TÉLUQ à I'UQAM. La poursuite du développement des CIF et leur intégration aux facultés est suspendue et 
la construction de l'université en ligne marque un " temps d'arrêt », malgré le fait qu'elle reste un objectif prioritaire inscrit dans le dernier plan TI de I'UQAM 2003-2006. Malgré ces aléas dans le processus de développement d'une université bimodale, des programmes de formation à distance ou en mode hybride se mettent en place, fruits d'un effort consacré par de petites équipes qui tentent de construire de nouvelles formes de collaboration entre les services administratifs et les instances pédagogiques. Ces changements sur les plans pédagogique, organisationnel et technologique suscitent un intérêt scientifique, notamment en ce qui concerne l'avenir des nouvelles formes d'encadrement pédagogique et de recherche aux cycles supérieurs.

\section{Le projet FODAR : « E-science, e-recherche et e-learning : nouvelles perspectives pour les études avancées ॥}

\section{Aspects théoriques et méthodologiques}

Consacré à l'étude de l'intégration des technologies et de l'adaptation des pratiques dans l'enseignement supérieur, le projet FODAR a démarré en novembre 2006. Réalisé en partenariat avec des chercheurs provenant de la TÉLUQ, de l'UQAM et de l'École de technologies supérieure (Université du Québec), le projet prend appui sur les concepts e-science, e-recherche et e-learning afin d'investir les problématiques et les pratiques d'enseignement et d'envisager l'usage des technologies dans les processus de formation et de recherche des programmes de deuxième et de troisième cycles offerts à distance ou en mode hybride et à l'international. Trois chantiers d'étude ont été mis en place afin de réaliser les objectifs énoncés, dans lesquels se reflètent trois dimensions complémentaires orientées vers la vision de la proximité :

1) L'appropriation d'une instrumentation technologique - ici la dimension technologique est axée sur la recherche et le choix d'une technologie appropriée et visant la proximité des personnes impliquées dans le processus (enseignants, étudiants, partenaires).

2) L'adaptation des pratiques pédagogiques de formation de cycles supérieurs - la relation pédagogique traditionnelle centrée sur le contenu se transforme et fait place à l'autonomie de l'apprenant et à la reconstruction du savoir par l'interaction sociale (étudiants, professeurs, pairs, environnement). L'analyse est donc centrée sur l'apprenant et ses compétences.

3) L'adaptation des pratiques de gestion académique - faut-il observer cette dimension selon une perspective constructiviste et socioconstructiviste, comme c'est le cas pour la dimension pédagogique?

En ce qui a trait au chantier de gestion académique, nous proposons d'observer celle-ci selon une vision centrée sur les " intéressés ": le concept des intéressés (théorie des intéressés de Freeman, 1984) montre qu'une organisation est perçue comme un ensemble de relations et de transactions entre tous les acteurs qui développent des « intérêts » pour l'organisation (dirigeants, clients, fournisseurs, citoyens). Ces acteurs intéressés sont autant ceux qui évoluent au sein de l'organisation, dans le contexte de la formation à distance (étudiant, professeur, tuteur, coordonnateur, superviseur), que ceux situés à l'extérieur (acteurs de la collaboration internationale). Nous considérons le fonctionnement d'une structure de gestion académique du programme comme une construction qui se réalise par la participation de divers acteurs " intéressés » qui partagent des pratiques et des savoirs et qui participent d'un même intérêt stratégique (réalisation de l'université virtuelle, mise en réseau de structures pédagogiques et de recherche). II s'agit alors d'identifier les différents " intéressés » qui interviennent dans la dimension de la gestion académique et de décrire leurs fonctions, leurs rôles et leurs interventions.

Visant les conditions d'adaptation d'un modèle de gestion académique pour des programmes de cycles supérieurs offerts à distance ou en mode hybride et international, les travaux effectués en 2006-2008 ont donc mis l'accent spécifiquement sur les pratiques de gestion académique. Cette recherche s'appuie sur l'analyse des discours institutionnels (documentation) et des études de cas, permettant ainsi l'observation du fonctionnement des programmes de formation à distance. La modélisation de ces pratiques est réalisée à partir de l'analyse des six programmes de formation supérieure de I'UQAM (4), de la TÉLUQ (1) et de l'Université de Montréal (1). La cueillette des données a été effectuée sur la base d'entretiens auprès de responsables et de coordonnateurs de programmes de cycles supérieurs (ceux offerts à distance ou en mode hybride et ceux en campus), et la modélisation des pratiques a été réalisée à partir du logiciel Mot+ du LICEF (TÉLUQ), en mettant en évidence les composantes du processus de gestion académique (les tâches, les acteurs, les ressources utilisées ou produites lors du fonctionnement).

\section{Les pratiques de gestion académique dans les programmes de formation supérieure : innovation organisationnelle et changement culturel}

D'une manière globale, nous relevons quatre types de programmes et de pratiques de gestion académique concernant la formation supérieure, qui seront analysés selon les modes de diffusion (en ligne et campus) et en fonction de leur structure d'appartenance, des tâches et des difficultés.

\section{Le type 1 - Programmes en ligne développés au sein de chaires de recherche (cas 1 et 2)}

Le programme court de deuxième cycle en ERE (éducation relative à l'environnement) est dispensé depuis 2003 sur campus et en ligne. Les étudiants sont admis continuellement dans le cas du programme en ligne. Le Ph.D. en sciences de l'éducation de l'Université de Montréal diffusé depuis 2006 en ligne est une adaptation du même programme campus existant depuis 1990. II est à signaler que le programme en ligne financé par l'Agence universitaire de la francophonie (AUF) ne concerne actuellement qu'une cohorte de 21 étudiants africains pour une période de trois années. Ce programme pourrait ne pas être renouvelé. 


\section{Exemple de modélisation des pratiques de gestion académique : le programme de $2^{\mathrm{e}}$ cycle en Éducation relative en environnement (ERE).}

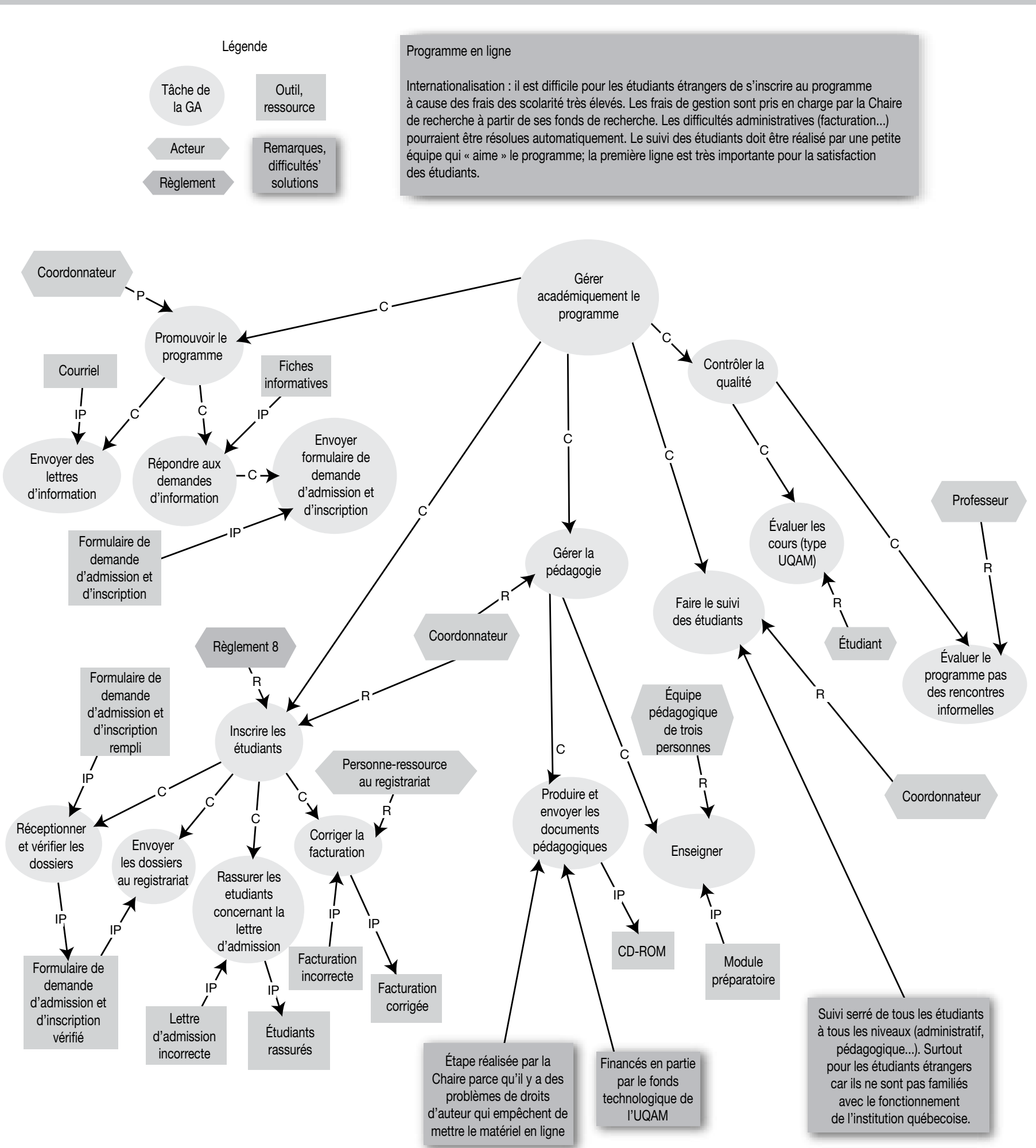


Les programmes en ligne développés au sein de chaires de recherche se caractérisent par :

- l'émergence d'une structure de gestion et d'encadrement ;

- leur prise en charge par une structure non dédiée à la gestion des programmes d'études supérieures.

D'une manière globale, ces programmes ont été créés, développés et gérés par des structures dédiées à l'activité de recherche : la chaire de recherche en ERE et la chaire de recherche du Canada sur les TIC en éducation. Au fil de l'expérience, ces chaires ont dû se doter de mécanismes pour suppléer aux lacunes du système de gestion institutionnel ou en corriger certains aspects qui n'arrivaient pas à satisfaire les besoins de tels programmes.

En raison de la nature de leurs activités, les chaires de recherche possèdent des caractéristiques qui les préparent à prendre en charge des enseignements supportés par les technologies et disposent ainsi :

- de structures souples et adaptables à différents contextes ;

- d'un personnel réduit capable d'assumer diverses fonctions reliées à l'intégration des technologies ;

- d'une pratique de négociation constante (recherche de subventions et de partenariats) ;

- d'une capacité de développer l'innovation et l'expérimentation ;

- d'une attitude réflexive par rapport à leurs propres pratiques (elles ont su transférer cette attitude à l'analyse des actions de formation à distance).

Ces caractéristiques leur permettent d'assurer la gestion académique de programmes en ligne et d'en améliorer les processus. Ce mode de fonctionnement nouveau constitue un contournement des structures administratives traditionnelles qui met en évidence une certaine inadaptation de ces dernières au mode de fonctionnement des programmes à distance. C'est pourquoi les chaires de recherche doivent prendre en charge la régulation du fonctionnement administratif de leurs programmes, en consacrant à la coordination administrative la moitié du temps dévolu à la coordination globale (gestion et encadrement). Ce mode d'organisation, en marge du fonctionnement habituel, s'avère lourd pour assurer le bon déroulement d'un programme de formation à distance.

La transformation structurelle et organisationnelle requise pour l'offre de programmes de formation à distance implique aussi des changements sur le plan de la culture organisationnelle. Les personnes interviewées ont fait état d'une certaine incompréhension entre le monde administratif et le monde de la pédagogie. Toutefois, dans les deux cas étudiés, les acteurs reconnaissent l'importance et la nécessité d'entretenir des relations de collaboration et de négociation entre les deux structures. Par exemple, un effort constant est fait pour sensibiliser l'administration à accepter des modifications en ce qui a trait à la révision des coûts d'inscription. Pourtant, on a relevé chez les personnes interviewées le sentiment que l'action s'inscrit encore et sans cesse dans une perspective " d'expérimentation ». Cela témoigne de la flexibilité des équipes de formation et de recherche, mais aussi de la difficulté de trouver un état de stabilité institutionnelle pour ce type de fonctionnement, même après plusieurs années d'existence. Les programmes à distance insérés dans les chaires mettent de l'avant des difficultés similaires : une grande charge de travail exigée par le suivi permanent de l'étudiant (pédagogique, administratif, socio-affectif), des frais de scolarité élevés qui affectent le nombre des inscriptions. Toutefois, il en ressort une grande motivation à évoluer dans un environnement qui stimule l'innovation continue et implique une capacité à faire le relais entre le monde pédagogique et le monde académique.

\section{Le type 2 - Les programmes campus (cas 3 et 4)}

II s'agit du programme de Ph.D. en muséologie, offert en partenariat international avec l'Université d'Avignon en France, et du programme de Ph.D. en sciences de l'environnement, qui est quant à lui un programme "réseau » de l'Université du Québec, offert en association avec les universités du Québec à Chicoutimi, à Rimouski, en AbitibiTémiscamingue et à Trois-Rivières (UQAC, UQAR, UQAT et UQTR). Des cours se donnent dans chaque université.

Ces programmes campus constituent un deuxième type qui se caractérise par la recherche d'un ajustement des structures traditionnelles de formation aux réalités des programmes en cotutelle (niveau international) ou en réseau (niveau provincial). Ils intègrent des technologies pour rejoindre les étudiants et permettre la réalisation des activités prescrites par les programmes : utilisation d'une plateforme pour le dépôt de documents (Ph.D. en sciences de l'environnement) ; utilisation de la vidéoconférence pour un cours à distance, l'examen de synthèse et le projet de thèse (Ph.D. en sciences de l'environnement) ; introduction de la vidéoconférence pour l'examen de synthèse et le projet de thèse (Ph.D. en muséologie) ; le courriel et le téléphone sont les outils de communication essentiels pour la gestion de ces deux programmes.

Les principales difficultés rencontrées par ces deux programmes résident dans l'ampleur de la charge de travail exigée pour en assurer le fonctionnement et dans la recherche de financement des étudiants. Par ailleurs, la multiplication des intervenants dans le cas des programmes "réseau » occasionne des problèmes de type relationnel qui impliquent la mise en œuvre d'une bonne collaboration et le partage de valeurs communes. Comme pour les programmes en ligne, les intervenants expriment un grand intérêt à évoluer dans un environnement aussi riche et innovant, et ce, malgré la charge de travail accrue.

\section{Le type 3 - Le programme International de MBA pour cadres (cas 5)}

Il s'agit d'un cas particulier de gestion académique d'un programme délocalisé dans plusieurs pays. Le programme est dispensé dans des universités partenaires à l'étranger. Ces universités partenaires gèrent la facturation des étudiants ainsi que la promotion du programme. Par contre, les cours sont dispensés par des professeurs de l'UQAM qui se déplacent à l'étranger (à l'exception de deux cours liés au contexte local). 
Si les problèmes de facturation et de frais de scolarité ne se posent pas comme dans les autres cas (facturation par les partenaires), la charge de travail consacrée au fonctionnement du programme se révèle importante, comme dans les autres programmes. On soulignera toutefois ici le problème lié au dégrèvement : on accorde seulement deux dégrèvements de cours alors que la charge réelle de travail imposée par le fonctionnement du programme s'avère, selon son responsable, nettement plus lourde (50 heures / semaine). II est important de considérer cet aspect de la gestion académique dans l'élaboration d'un modèle performant de fonctionnement de la formation à distance. L'analyse d'un tel programme, qui n'est pas en ligne, à l'instar des programmes de type 2 campus, nous renseigne toutefois sur les pratiques mises en œuvre dans les partenariats internationaux.

\section{Le type 4 - Le programme de maîtrise en FAD (maîtrise en formation à distance) (TÉLUQ) (cas 6)}

Le cas 6, maîtrise en formation à distance de la TÉLUQ, est un programme en ligne qui bénéficie d'une structure orientée totalement vers la formation à distance. Sur le plan des ressources, cette structure est organisée pour répondre aux besoins de conception, de production et de diffusion des programmes de formation supérieure à distance. L'analyse du cas a mis en évidence des pratiques de gestion académique centrées sur l'étudiant et mises en œuvre par une équipe qui développe la culture nécessaire au bon fonctionnement des programmes : collaboration étroite entre les membres et partage de valeurs communes. En effet, la gestion académique d'un programme de deuxième cycle à distance est perçue comme devant être un processus basé sur la prévention des problèmes et des conflits et sur la rapidité à les résoudre, notamment par un processus collaboratif.

L'existence actuelle à l'UQAM de telles pratiques permet d'entrevoir la possibilité de mettre en œuvre un processus de gestion académique plus adapté à la formation en ligne et à distance. Cette perspective nécessitera un changement culturel, celui lié à la perception de cette modalité de formation, notamment celle que la TÉLUQ pratique, ainsi que la perception de son rôle au sein du processus de développement de I'UQAM comme université bimodale.

\section{Conclusion}

L'analyse des études de cas de programmes, notamment ceux en ligne, et leur modélisation nous permettent de comprendre le fonctionnement réel de la gestion académique et de la gestion administrative d'un programme de formation à distance. Malgré l'échantillon réduit des cas de programmes en ligne, les données recueillies nous permettront de faire émerger les composantes liées à un modèle performant de la formation à distance ou en mode hybride, notamment en tourisme. La formation en tourisme se trouve dans un contexte technologique et scientifique favorable : d'une part, l'importance d'une adaptation accrue des TIC dans l'enseignement supérieur et, d'autre part, l'émergence de nouvelles approches dans la recherche sociale, telle la transdisciplinarité suscitée par la complexité de la réalité à observer (en tourisme par exemple) (Kadri et Bédard, 2006). Cette conjonction offre à la formation en tourisme une base stratégique pour la diffusion des connaissances et le développement d'un partenariat interuniversitaire international visant à renforcer la recherche de nouvelles pratiques d'enseignement et de formation à la recherche en mode hybride (en classe et à distance). Le projet de développement d'un programme de doctorat en sciences du tourisme s'inscrit dans ce contexte de l'innovation dans la recherche sociale.

Boualem Kadri est professeur au Département d'études urbaines et touristique de l'Université du Québec à Montréal.

François Bédard est professeur au Département d'études urbaines et touristiques de l'Université du Québec à Montréal. II est également directeur du Centre international de formation et de recherche en tourisme (CIFORT).

\section{Notes}

1 Le projet FODAR, coordonné par France Henri (TÉLUQ), est réalisé en partenariat, auquel participent : François Bédard, Ghislain Lévesque, Boualem Kadri (UQAM) et Nicola Hagemeister (ETS).

2 Les résultats de cette étude ont fait l'objet d'un rapport d'étape diffusé en ligne (Bédard et Géronimi, 2002) et d'un article intitulé : "Les enjeux de l'intégration des TIC à l'Université : adaptation du corps enseignant et transformation des pratiques d'enseignement. L'expérience de l'Université du Québec à Montréal » (Bédard et al., 2005).

3 Vice-rectorat aux études.

\section{Bibliographie}

Bédard, François et Martine Géronimi (2002). L'ajustement aux technologies de l'information et de la communication : stratégies émergeant des attitudes et des comportements des enseignantes et des enseignants universitaires, Rapport d'étape sur l'enquête menée au printemps 2002 auprès du corps enseignant de I'UQAM. Document accessible sur le site Internet du Laboratoire en technologies de l'information et de la communication pour l'enseignement (LABTIC), [http://www.unites. uqam.ca/LABTIC/publications.htm].

Bédard, François, Boualem Kadri, Patrick Giroux, Martine Géronimi, Benoît Duguay et Danièle Boulard (2005), "Les enjeux de l'intégration des TIC à l'Université : adaptation du corps enseignant et transformation des pratiques d'enseignement. L'expérience de I'Université du Québec à Montréal ", Revue RES ACADEMICA de l'Association internationale de pédagogie universitaire (AIPU), vol. 23, n² 2, p. 7025.

Comité de mise en œuvre du rattachement de la Télé-université à l'UQAM (2006), Cadre de gestion expérimental du plan de développement de la formation à distance à l'UQAM, Montréal, UQAM.

CREPUQ (Conférence des recteurs et des principaux des universités du Québec) (1999), Université - An 2010. Mise en valeur des technologies de l'information et des communications pour la formation universitaire, Secrétariat de la CREPUQ, Québec.

Freeman, E.R. (1984), Strategic Management: A Stakeholder Theory Approach. USA, Boston and Toronto, Pitman.

Kadri, Boualem, et François Bédard (2006), «Vers une science du tourisme? Complexité et transdisciplinarité ", Téoros, vol. 25, n 1, p. 62-64.

Karsenti, Thierry et François Larose (2001), Les TIC au cœur des pédagogies universitaires, Québec, Presses de l'Université Laval.

UQAM-Comité conseil conjoint VRAE-VRSADT (2003), Appropriation des technologies de l'information dans la formation à l'UQAM. 